\title{
Chimeras confined by fractal boundaries in the complex plane
}

\author{
Ralph G. Andrzejak ${ }^{a}$ \\ Universitat Pompeu Fabra, Department of Information and Communication Technologies, 08018 Barcelona, \\ Catalonia, Spain
}

(Dated: 6 May 2021)

Complex-valued quadratic maps either converge to fixed points, enter into periodic cycles, show aperiodic behaviour or diverge to infinity. Which of these scenarios takes place depends on the map's complex-valued parameter $c$ and the initial conditions. The Mandelbrot set is defined by the set of $c$ values for which the map remains bounded when initiated at the origin of the complex plane. In this study we analyze the dynamics of a coupled network of two pairs of two quadratic maps in dependence on the parameter $c$. Across the four maps, $c$ is kept the same whereby the maps are identical. In analogy to the behavior of individual maps, the network iterates either diverge to infinity or remain bounded. The bounded solutions settle into different stable states, including full synchronization and desynchronization of all maps. Furthermore, symmetric partially synchronized states of within-pair synchronization and across-pair synchronization as well as a symmetry broken chimera state are found. The boundaries between bounded and divergent solutions in the domain of $c$ are fractals showing a rich variety of intriguingly esthetic patterns. Moreover, the set of bounded solutions is divided into countless subsets throughout all length scales in the complex plane. Each individual subset contains only one state of synchronization and is enclosed within fractal boundaries by $c$ values leading to divergence.

This work is dedicated to the late Vadim Anishchenko. His landmark papers on maps and on chimera states in networks of coupled maps ${ }^{1-18}$ represent only one branch in his plentiful contributions to the fields of nonlinear dynamics of deterministic and stochastic systems. Chimera states are characterized by the coexistence of synchronization and desynchronization in networks of coupled dynamics ${ }^{19,20}$. The set of initial conditions leading to chimera states can form riddled and fractal basins of attraction ${ }^{21-23}$. Initial conditions outside of these basins of attraction can result in fully synchronized or fully desynchronized dynamics, among others. While chimera states were first described ${ }^{19}$ and continue to be studied for networks of time-continuous oscillators, an increasing interest is paid to networks of time-discrete maps. Chimera states were found for networks of coupled logistic maps ${ }^{4-9,23-31}$, Henon maps ${ }^{10-15,21}$, cubic maps ${ }^{7,16,17}$, map-based neuron models ${ }^{18,32}$, sine-circle maps ${ }^{33-35}$, sine-squared $\operatorname{maps}^{23,26,36}$, $\operatorname{cosine}_{\mathbf{m a p s}}{ }^{37}$, piecewise linear maps ${ }^{38}$, and piecewise logistic maps ${ }^{38}$. Recently, two populations of quadratic maps with real-valued parameter $c$ were shown to yield a plentitude of dynamics including chimera states ${ }^{39}$. Quadratic maps, however, reveal their full complexity only for complexvalued parameters $c$. In particular, they allow one to generate fractal Julia sets and Mandelbrot sets in the complex plane ${ }^{40-44}$. Our aim here is to explore this complexity without adding the complicatedness of a high-dimensional network. We therefore study a minimal two-population network of two pairs of two quadratic maps with complex-valued parameters $c$. We use non-random initial conditions defined by the first four iterates of uncoupled quadratic maps. Our results show that in dependence on $c$, the network iterates can diverge to infinity or remain bounded, and that bounded solutions can get fully synchronized, fully desynchronized, or enter into some partially synchronized state, including a symmetry broken chimera state. The boundaries between these different states in the domain of the complex-valued $c$ form fractal patterns. In contrast to the high-dimensional fractal basins of attraction $^{21-23}$, these fractals reside in the complex plane and are therefore straightforward to visualize. In qualitative terms, the fractals generated by our network of coupled quadratic maps seem less filigree, more disordered, but even fuller of variety than the Mandelbrot set. Complementing previous work $^{21-23}$, our study establishes an intriguing link between the dynamics of partially synchronized networks and the geometry of fractals in the complex plane.

\footnotetext{
a)Electronic mail: ralph.andrzejak@upf.edu (Author to whom correspondence should be addressed). Citation: Chaos 31, 053104 (2021); doi: 10.1063/5.0049631
} 


\section{INTRODUCTION}

We use the quadratic map $f_{c}\left(z_{n+1}\right)=z_{n}^{2}+c$, where $n$ is discrete time in units of the iteration step, and $c$ is a complex-valued parameter. The complex-valued iterates of $f_{c}$ either remain bounded for all times $n$ or diverge to infinity. Whether or not the iterates diverge depends on the parameter $c$ and the initial condition $z_{0}$. Given $f_{c}$ and a certain $c$, the Julia set is defined by the boundary between the complex-valued initial conditions for which $f_{c}$ diverges and the ones for which it remains bounded ${ }^{40,41}$. If and only if the origin of the complex plane $z_{0}=0$ belongs to the initial conditions which lead to bounded behavior of $f_{c}$, the Julia set is connected. Otherwise, the Julia set is a Cantor space. The Mandelbrot set is defined by the set of $c$ for which the Julia set is connected, or equivalently, by the set of $c$ for which iterates $z_{n}$ of $f_{c}$ initiated at $z_{0}=0$ remain bounded for all $n .^{42-44}$

We have shown previously that two identical populations of quadratic maps $f_{c}$ yield a plentitude of dynamics which can be symmetric or symmetry broken with regard to the two populations ${ }^{39}$. In particular, these dynamics included chimera states for which one population was identically synchronized while the other remained incoherent. However, in this previous work we only considered real-valued parameters $c$ and initial conditions, such that the dynamics of the populations remained in the real-valued domain. What type of dynamics can arise if complex-valued parameters $c$ are used? To address this question, we here analyze a coupled network $F_{c}$ of two pairs, each composed by two quadratic maps $f_{c}$, which is the smallest network that allows us to study synchronization within and across pairs of maps. This parsimonious approach to use a minimal two-population network of only two maps per population is different from Ref. 39, where each population consisted of one hundred maps. For previous work on chimera states in networks consisting of a minimal number of nodes see for example Refs. $45-52$.

\section{A MINIMAL TWO-POPULATION MODEL}

The map $f_{c}$ is governed by a sole parameter $c$, which is set to be the same for all four maps of the network $F_{c}$. Hence, all maps of $F_{c}$ are identical. Each map is coupled with strength $C_{\mathrm{w}}$ to the other map within its own pair and with strength $C_{\mathrm{a}}$ to the two maps in the other pair. The maps of the first pair are called $U_{c}$ and $V_{c}$, and the ones of the second pair are called $P_{c}$ and $Q_{c}$. Accordingly, $F_{c}$ can be written as:

$$
\begin{array}{cc}
U_{c}: & u_{n+1}=f_{c}\left(u_{n}\right)+C_{\mathrm{W}} \cdot\left[f_{c}\left(v_{n}\right)-f_{c}\left(u_{n}\right)\right]+C_{\mathrm{a}} \cdot\left[\left(f_{c}\left(p_{n}\right)-f_{c}\left(u_{n}\right)\right)+\left(f_{c}\left(q_{n}\right)-f_{c}\left(u_{n}\right)\right)\right] \\
V_{c}: & v_{n+1}=f_{c}\left(v_{n}\right)+C_{\mathrm{W}} \cdot\left[f_{c}\left(u_{n}\right)-f_{c}\left(v_{n}\right)\right]+C_{\mathrm{a}} \cdot\left[\left(f_{c}\left(p_{n}\right)-f_{c}\left(v_{n}\right)\right)+\left(f_{c}\left(q_{n}\right)-f_{c}\left(v_{n}\right)\right)\right] \\
P_{c}: & p_{n+1}=f_{c}\left(p_{n}\right)+C_{\mathrm{W}} \cdot\left[f_{c}\left(q_{n}\right)-f_{c}\left(p_{n}\right)\right]+C_{\mathrm{a}} \cdot\left[\left(f_{c}\left(u_{n}\right)-f_{c}\left(p_{n}\right)\right)+\left(f_{c}\left(v_{n}\right)-f_{c}\left(p_{n}\right)\right)\right] \\
Q_{c}: & q_{n+1}=f_{c}\left(q_{n}\right)+C_{\mathrm{W}} \cdot\left[f_{c}\left(p_{n}\right)-f_{c}\left(q_{n}\right)\right]+C_{\mathrm{a}} \cdot\left[\left(f_{c}\left(u_{n}\right)-f_{c}\left(q_{n}\right)\right)+\left(f_{c}\left(v_{n}\right)-f_{c}\left(q_{n}\right)\right)\right]
\end{array}
$$

Eqs. (1-4) are the usual equations for two populations of coupled maps. For such a small network, we can use a different symbol for each map instead of indices, which allows us to simplify the notation below. We study $F_{c}$ in dependence on the parameter $c$ for the following non-random initial conditions. For every value of $c$, we calculate the first four iterates of the individual quadratic map $f_{c}$ started at $z_{0}=0$. These values are then used as initial conditions of the four coupled maps: $u_{0}=z_{1}, v_{0}=z_{2}, p_{0}=z_{3}, q_{0}=z_{4}$. Therefore, except for $c$ values for which $f_{c}$ directly enters into a fixed point, period- 2 or period-3 cycle the network is always initialized in a fully asynchronous state at time $n=0$. We run the network for a total of $n_{\max }=5 \times 10^{5}$ iterations. This choice of $n_{\max }$ and why we do not use random initial conditions is discussed in detail below. Unless stated otherwise, the coupling strengths are fixed at $C_{\mathrm{W}}=0.01$ and $C_{\mathrm{a}}=0.0025$. These parameters are set such that the coupling within pairs is stronger than the one across pairs. Beyond this constraint the exact values of $C_{\mathrm{w}}$ and $C_{\mathrm{a}}$ are not tuned in any way.

If for a certain $c$, the maps leave the range of floating point numbers, we determine the onset of this divergence by $n_{\text {div }}=\min \left\{n \mid \max \left\{\left|u_{n}\right|,\left|v_{n}\right|,\left|p_{n}\right|,\left|q_{n}\right|\right\}>2\right\}$, where $|\cdot|$ denotes the absolute value in the complex plane. This threshold is arbitrary, and the particular value of 2 is taken over from the point-of-no-return criterion of individual maps $f_{c}$ (see also Section VI). For all bounded solutions, we use the following criteria to test for synchronization between all six pairings of maps and to determine if the maps have entered into a periodic cycle. To detect synchronization between the maps $U_{c}$ and $V_{c}$, we use the criterion $\left|u_{n_{\max }}-v_{n_{\max }}\right|<\varepsilon_{\mathrm{NUM}}=10^{-13}$, which corresponds to identical synchronization within the limits of numerical precision ${ }^{53}$. We write $U_{c}=V_{c}$ to indicate that $U_{c}$ and $V_{c}$ are synchronized, and $U_{c} \neq V_{c}$ to indicate that they are not. The same criterion and analogous notations are used for all five remaining pairings of maps. To detect a period- $m$ cycle of the map $U_{c}$, we use the criterion $\left|u_{n_{\max }}-u_{n_{\max }-m}\right|<\varepsilon_{\mathrm{NUM}}$, and analogously for $V_{c}, P_{c}$, and $Q_{c}$. We never observed dynamics for which the different maps were in periodic cycles of different lengths. Accordingly, we directly refer to a period- $m$ cycle of the network $F_{c}$. A fixed point of $F_{c}$ corresponds to $m=1$. 

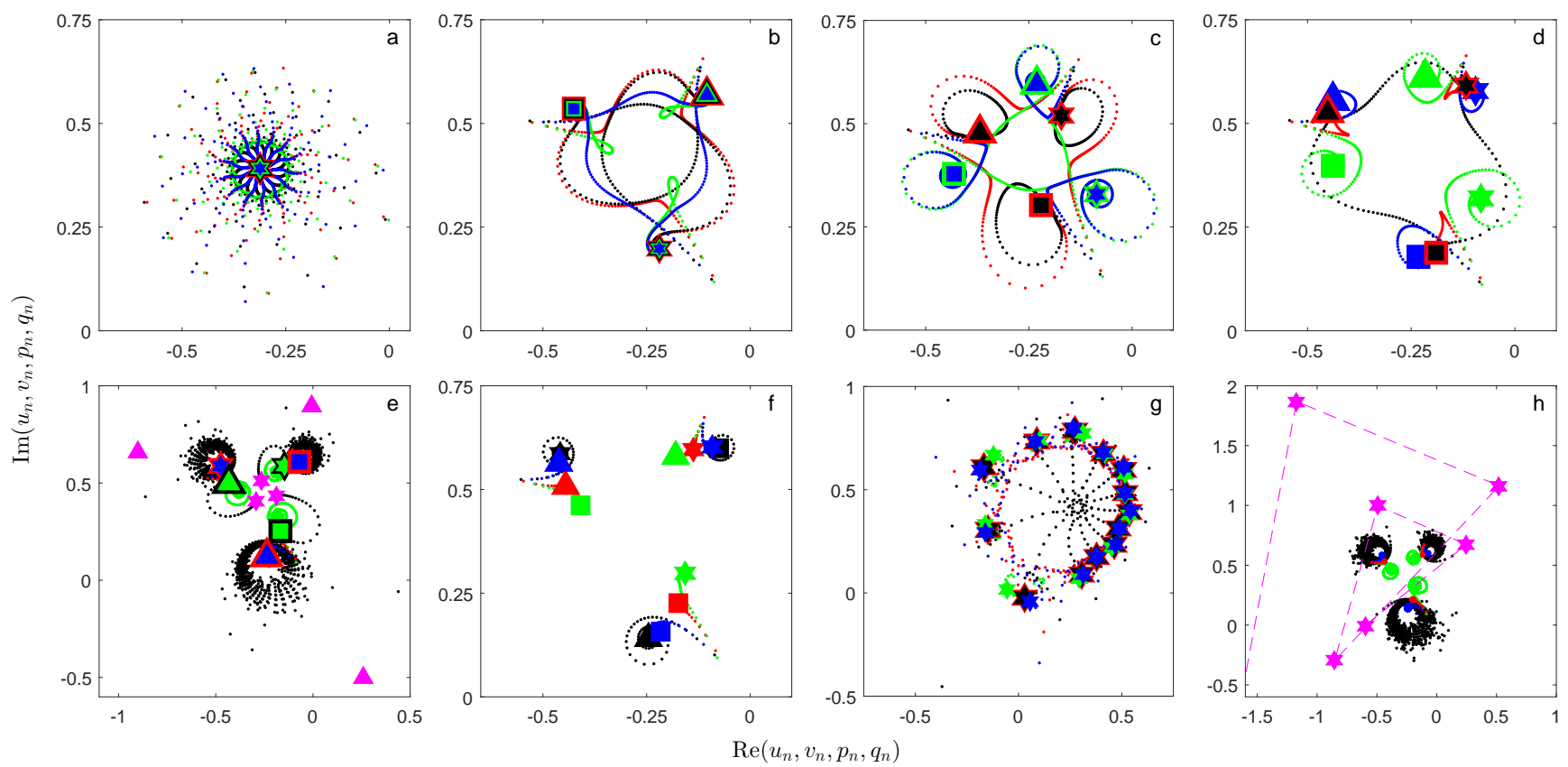

FIG. 1. Different values of $c$ lead to qualitatively different dynamics of the network of four coupled maps $F_{c}$. Iterates $u_{n}$ (red), $v_{n}$ (black), $p_{n}$ (green), and $q_{n}$ (blue) obtained for eight exemplary values $c_{1, \ldots, 8}$, listed in table II in the Appendix. (a) Results for $c_{1}$. At $n=18599$, all four maps have converged to a joint fixed point. Small dots: iterates during initial transients, $n=1, \ldots, 18598$. Stars: $u_{n}=v_{n}=p_{n}=q_{n}$ for $n \geq 18599$. Stars of different sizes allow all overlaid symbols to be seen. Panel (b) is analogous to panel (a) but for $c_{2}$. Here convergence to $u_{n}=v_{n}=p_{n}=q_{n}$ is reached at $n=1487$. Additional symbols are used because the maps converge to a period-3 cycle. Triangles: $n=1487,1490, \ldots ;$ squares: $n=1488,1491, \ldots$; stars $n=1489,1492, \ldots$. (c) For $c_{3}$, convergence to $u_{n}=v_{n}$ and $p_{n}=q_{n}$ is reached at $n=6299$. Symbols continue to indicate the different phases of the period-3 cycles. Since the maps synchronize within pairs but not across pairs, only the red and black as well as the blue and green symbols are overlaid. (d) Convergence to the chimera state of $u_{n}=v_{n}$ and $p_{n} \neq q_{n}$ is reached at $n=2318$ for $c_{4}$. (e) For $c_{5}$ convergence to $u_{n}=q_{n}$ and $v_{n}=p_{n}$ is reached at $n=6175$. Additional magenta symbols serve to highlight the abrupt reorganization of the dynamics. Magenta triangles: $u_{n}$ for $n=4758,4759,4760$; magenta stars: $u_{n}$ for $n=4761,4762,4763$. (f) For $c_{6}$ convergence is attained at $n=2934$. Here all maps converge to a distinct period-3 cycle such that no symbols are overlaid. (g) Like for $c_{4}$ in panel (d), for $c_{7}$ the dynamics converges to $u_{n}=v_{n}$ and $p_{n} \neq q_{n}$, here at $n=1749$. Instead of a period-3 cycle, we find a period-13 cycle. Stars depict all 13 phases of these cycles, which are visited clock-wise advancing two positions per iteration such that after 13 iterations the ring-shaped structure is circumscribed twice. (h) For $c_{8}$ the dynamics does not settle to any bounded solution but diverges towards infinity. Magenta stars connected by lines show the iterates $u_{n}$ for $n=8336, \ldots, 8341$. The iterate $u_{8342}$ is already outside of the displayed range. Ten iterations later $u_{n}, v_{n}, p_{n}, q_{n}$ will all have left the range of floating point numbers.

\section{THE DYNAMICS IN THE COMPLEX DOMAIN OF THE NETWORK ITERATES}

Figure 1 shows the dynamics of $F_{c}$ obtained for eight exemplary $c$ values. We denote these values by $c_{1, \ldots, 8}$ and list them in table II in the Appendix. In the first example $\left[c_{1}\right.$, Fig. 1(a)], the four maps undergo different transient states each, but then they all converge to the same fixed point, i.e. we get $U_{c}=V_{c}=P_{c}=Q_{c}$. We also obtain this full synchronization of all maps for $c_{2}$ [Fig. 1(b)]. However, in this case the maps do not converge to a fixed point but jointly enter into a period- 3 cycle. Also in the third example $\left[c_{3}\right.$, Fig. $\left.1(\mathrm{c})\right], F_{c}$ enters into a period- 3 cycle. In contrast to the first two examples, however, synchronization is found within the two pairs but not across them. That means we get within-pair synchronization, $U_{c}=V_{c} \neq P_{c}=Q_{c}$. A chimera state is established in the fourth example [c $c_{4}$, Fig. 1(d)]. While the first pair synchronizes $\left(U_{c}=V_{c}\right)$, the maps of the second pair enter into distinct cycles and remain unsynchronized among them $\left(P_{c} \neq Q_{c}\right)$ and also from the synchronized pair $\left(P_{c} \neq U_{c}=V_{c} \neq Q_{c}\right)$. A somewhat counter-intuitive result is obtained in the next example $\left[c_{5}\right.$, Fig. $\left.1(\mathrm{e})\right]$. Here the maps do not synchronize within populations but across populations. All maps at first follow their respective transient motions. For example, the iterates of $V_{c}$ initially fill three ring-shaped structures in an aperiodic way. Then at $n \approx 4760$, the dynamics reorganizes abruptly. The map $V_{c}$ is driven away from its initial ring-shaped transients and then re-injected to the center of the overall dynamics. Instead of returning to the initial ring-shaped structures it then synchronizes with $P_{c}$. Furthermore, $U_{c}$ and $Q_{c}$ synchronize across pairs. Overall, we get $U_{c}=Q_{c} \neq V_{c}=P_{c}$, i.e. across-pair synchronization 


\begin{tabular}{|lll|l|l|}
\hline Type & Description & Formula & $\begin{array}{l}\text { Example } \\
\text { in Fig. 1 }\end{array}$ & $\begin{array}{l}\text { Color in } \\
\text { Figs. 3-6(a) }\end{array}$ \\
\hline \hline Full synchronization & $\begin{array}{l}\text { All maps } \\
\text { synchronized }\end{array}$ & $U_{c}=V_{c}=P_{c}=Q_{c}$ & (a), (b) & blue \\
\hline Within-pair synchronization & $\begin{array}{l}\text { Maps synchronized within pairs but } \\
\text { desynchronized across pairs }\end{array}$ & $U_{c}=V_{c} \neq P_{c}=Q_{c}$ & (c) & yellow \\
\hline Across-pair synchronization & $\begin{array}{l}\text { Maps synchronized across pairs but } \\
\text { desynchronized within pairs }\end{array}$ & $\begin{array}{l}U_{c}=P_{c} \neq V_{c}=Q_{c} \text { or } \\
U_{c}=Q_{c} \neq V_{c}=P_{c}\end{array}$ & (e) \\
\hline Chimera state & $\begin{array}{l}\text { Maps synchronized in one pair but } \\
\text { desynchronized in the other pair }\end{array}$ & $\begin{array}{l}U_{c}=V_{c} \text { but } P_{c} \neq Q_{c} \text { or } \\
P_{c}=Q_{c} \text { but } U_{c} \neq V_{c}\end{array}$ & green \\
\hline Full desynchronization & $\begin{array}{l}\text { All pairings of maps (g) } \\
\text { desynchronized }\end{array}$ & $\begin{array}{l}U_{c} \neq V_{c}, V_{c} \neq P_{c}, P_{c} \neq Q_{c}, \\
Q_{c} \neq U_{c}, Q_{c} \neq V_{c}, P_{c} \neq U_{c}\end{array}$ & (f) \\
\hline
\end{tabular}

TABLE I. Five states of synchronization illustrated in Figs. 1(a)-1(g). The penultimate column indicates the panels of Fig. 1 which contain the corresponding examples. The last column specifies the colors used to represent the states in Figs. 3-6(a). Recall that we use the equality sign to indicate that the maps are identically synchronized within the limits of numerical precision.

instead of within-pair synchronization. In the sixth example $\left[c_{6}\right.$, Fig. $\left.1(f)\right]$, none of the maps synchronize. Instead each map enters into a distinct period-3 cycle. Inspecting the spatial distribution of the three different symbols that represent the phase of the maps within their respective cycle, we see that maps follow similar cycles but with a relative lag to each other. This lag becomes most evident for the example of $c_{6}$, but can also be seen for $c_{4}$ and $c_{5}$. In the examples discussed so far, we used a fixed point in Fig. 1(a) and period-3 cycles in Figs. 1(b)-1(f) simply for ease of readability of these figures. However, all states of synchronization can also be attained for higher order periods, as illustrated by a chimera state of period $13\left[c_{7}\right.$, Fig. $\left.1(\mathrm{~g})\right]$. Like in the fourth example in Fig. 1(d), the pair $U_{c}$ and $V_{c}$ synchronizes identically, while the maps of the pair $P_{c}$ and $Q_{c}$ enter into distinct cycles and remain unsynchronized. The five different states of synchronization seen in the examples of Figs. 1(a)-1(g) are summarized in table I.

The parameter value used for the last example $c_{8}$ in Fig. $1(\mathrm{~h})$ is obtained by only slightly changing $c_{5}$. Both values differ by $10^{-7}$ in their imaginary part (see again table II in the Appendix). As a result of the smallness of this change, the initial transients obtained for $c_{8}$ look similar to those obtained for $c_{5}$. However, eventually a different outcome is obtained. At $n \approx 8340$ the map $V_{c}$ gets carried away from its initial ring-shaped transients. This is similar to what happens for $c_{5}$ after approximately 4750 iterations. However, for $c_{8}$ the iterates of $V_{c}$ are not re-injected to the center of the overall dynamics but diverge towards infinity instead. As a consequence of the coupling also the other maps get carried away towards infinity. By the time of iteration $n=8352$, the iterates of all maps have left the range of floating point numbers. In contrast to all previous examples $c_{1, \ldots, 7}$, the network $F_{c}$ diverges for $c_{8}$. Hence, the dichotomous behaviour of $f_{c}$, of either remaining bounded or diverging, carries over to our network of four coupled maps $F_{c}$.

We can now relate the value of $n_{\max }$ fixed above to the length of the transients observed in Fig. 1 . The network $F_{c}$ always undergoes transients before converging to some final bounded state or diverging to infinity. We, therefore, have to estimate the number of iterations for which we can assume that $F_{c}$ has settled to its asymptotic dynamics. The problem is that the length of the transients depends on $c$. Any bounded or divergent dynamics can be preceded by short or long transients. Moreover, for some $c$ values the transients can be longer than times accessible for numerical simulations. Being aware of this unavoidable limitation, we determined in pre-analysis that the value $n_{\max }=5 \times 10^{5}$ leads to a sufficient precision throughout all ranges of $c$ we consider below.

\section{MAPPING THE ASYMPTOTIC DYNAMICS IN THE COMPLEX DOMAIN OF THE PARAMETER $c$}

Results of Fig. 1 show that different values of $c$ can lead to qualitatively different dynamics of $F_{c}$. Even an arbitrarily small difference in $c$ can be decisive for the outcome [see again Fig. 1(e) versus Fig. 1(h)]. What results are obtained when we vary $c$ quasi-continuously in the complex plane? To address this question, we look at mappings of bounded versus divergent behaviour of $F_{c}$. In analogy to the definition of the Mandelbrot set, we define $\mathcal{A}$ by the set of $c$ values for which the network $F_{c}$ shows bounded behaviour [such as in Figs. 1(a)-1(g)]. The complement of $\mathcal{A}$ is called $\mathcal{B}$. In other words, $\mathcal{B}$ is the set of $c$ for which the network shows divergent behaviour [such as in Fig. 1(h)]. The set $\mathcal{A}$ is divided further into subsets with regard to the different periodic cycles or the different states of synchronization attained by the iterates of $F_{c}$ as a function of $c$.

Figures 2(a) and 2(b) allow comparing the macroscopic shape of $\mathcal{A}$ to the one of the Mandelbrot set and inspecting the division of both sets with regard to the periodic cycles of the respective dynamics $F_{c}$ and $f_{c}$. While alterations 

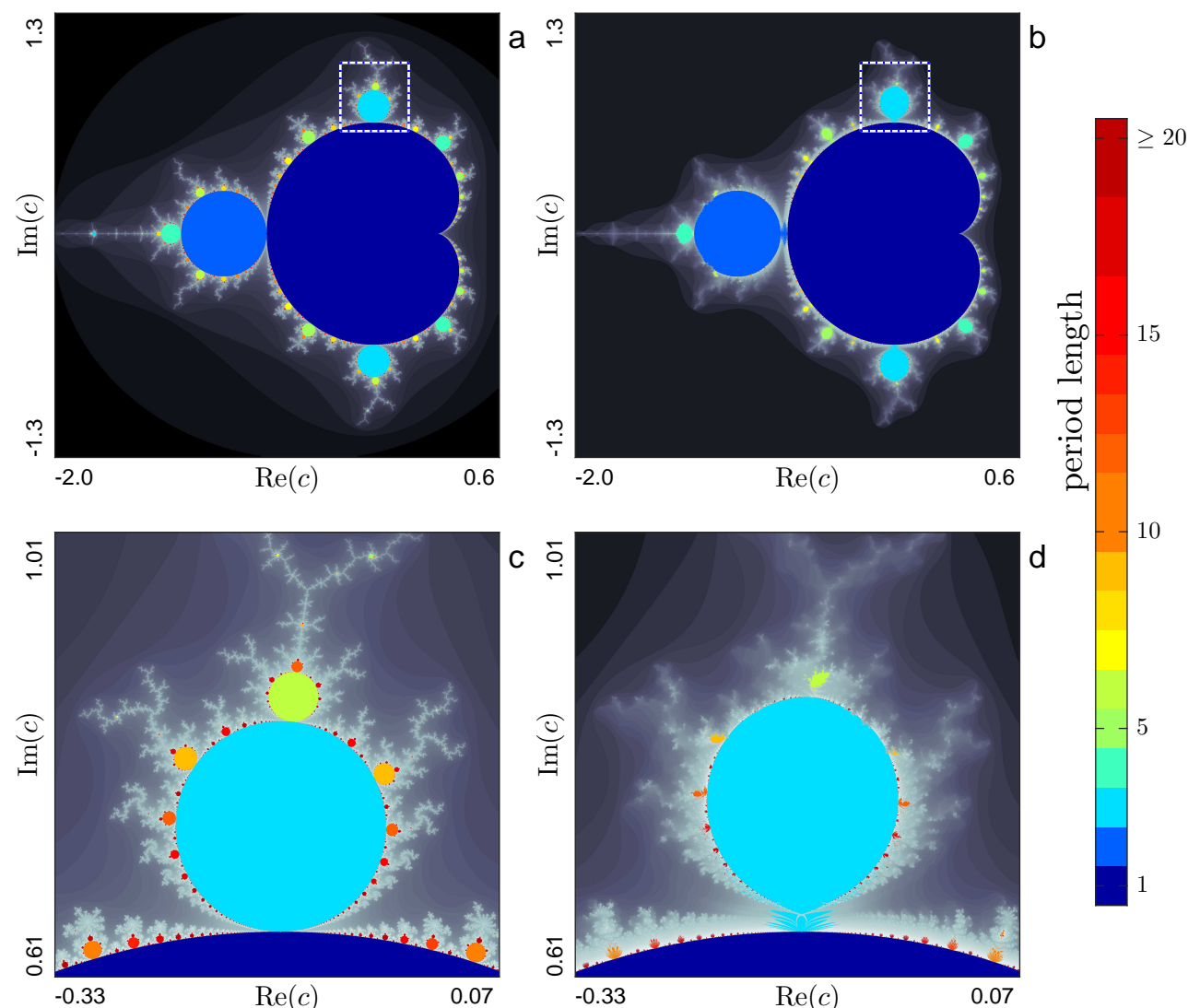

FIG. 2. The Mandelbrot set's overall morphology and spatial organization of periodic cycles is preserved in $\mathcal{A}$. (a) Gray areas: complement of the Mandelbrot set, i.e. the set of complex $c$ for which the iterates of an individual quadratic map $f_{c}$ diverge to infinity. The Mandelbrot set is given by the union of all non-gray areas. Inside the Mandelbrot set, the color indicates the period of the cycle into which the map $f_{c}$ enters. (b) Gray areas: complement of the set $\mathcal{A}$, i.e. the set $\mathcal{B}$ of complex $c$ for which the iterates of the network of four coupled quadratic maps $F_{c}$ diverge to infinity. Inside $F_{c}$, the color indicates the period of the cycle into which the network $F_{c}$ enters. Panel (c) and (d) magnify the dashed squares in panel (a) and (b), respectively. In all panels, values of $c$ are sampled on a regular $2000 \times 2000$ grid, and axes labels indicate values of the real and imaginary part of $c$ in the outermost corners. The gray scales are based on $\log _{10} n_{\text {div }}$, where lighter gray indicates higher $n_{\text {div }}$. The scales are adjusted for each panel to optimize the contrast.

can be seen in the peripheral structure, $\mathcal{A}$ inherits the overall morphology from the Mandelbrot set, including the symmetry around the real axis. Furthermore, the following well-known regularities in which periodic cycles are organized within the Mandelbrot set ${ }^{54}$ are preserved for $\mathcal{A}$. For $c$ values in the main cardioid both $f_{c}$ and $F_{c}$ converge to fixed points. For the first main bulb positioned on the left side of the main cardioid, the dynamics are attracted by period-2 cycles. The subsequent bulb on the left has period- 4 . The upper-most and lower-most bulbs adjacent to the main cardioid are of period-3. To locate these period-3 bulbs, note that the upper ones are included in the dashed squares in Figs. 2(a) and 2(b). Starting from these period-3 bulbs and going rightwards along the rim of the main cardioid, the locally largest bulbs are of period $4,5,6$, and so on. On the left side of the period-3 bulbs, the locally largest bulbs have period 5, 7, 9, and so on. The periods of smaller bulbs on the rim of the main cardioid which are not included in these sequences of locally largest bulbs can be determined as follows. For any pair of bulbs, the period of the biggest bulb between them is given by the pair's sum of periods.

Notwithstanding the aforementioned common overall morphology of $\mathcal{A}$ and the Mandelbrot set, the bulbs of $\mathcal{A}$ seem compressed, deformed, or missing altogether [Figs. 2(a) and 2(b)]. That these alterations do not only affect the shape of the bulbs becomes evident once we take a closer look at the upper principal period-3 bulb as an example [Figs. $2(\mathrm{c})$ and $2(\mathrm{~d})$ ]. For $\mathcal{A}$, the connection between this light blue bulb and the dark blue main cardioid is obstructed by gray ridges belonging to $\mathcal{B}$. Furthermore, pronounced changes are seen in the peripheral structure of the bulb. The filaments surrounding the bulb in the Mandelbrot set seem torn apart in $\mathcal{A}$. Does this mean that $\mathcal{A}$ is merely a distorted and corrupted Mandelbrot set? 

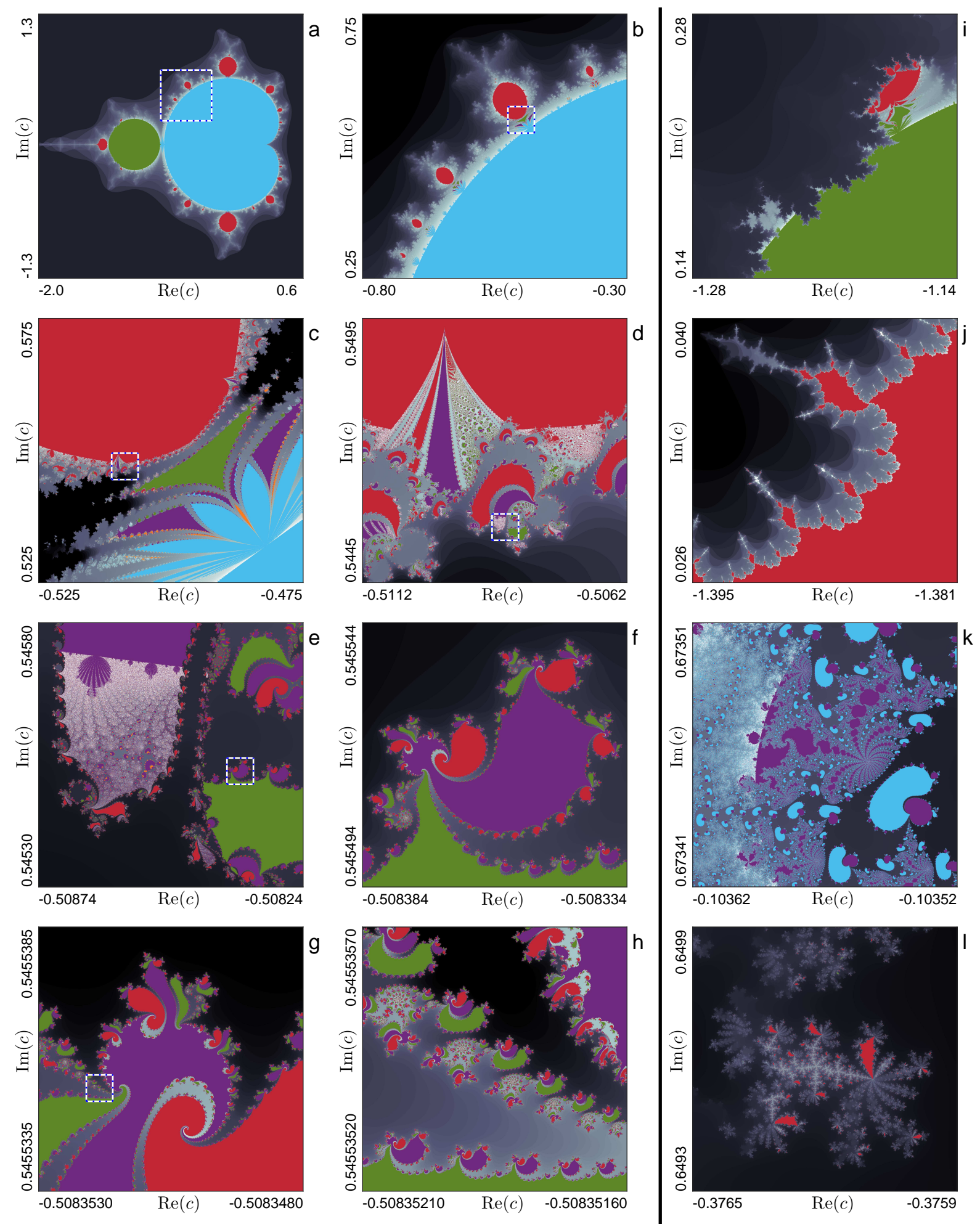

FIG. 3. Vadim's flowers and strawberry fields. In all panels, gray colors show the set $\mathcal{B}$, i.e. the set of complex $c$ for which the iterates of the network of four coupled quadratic maps $F_{c}$ diverge. Values of $c$ constituting $\mathcal{A}$ are depicted in different non-gray colors, each of which corresponds to a different state of synchronization. Blue: full synchronization; red: full desynchronization; yellow: within-pair synchronization; green: across-pair synchronization; purple: chimera state. Panels (b)-(h) are successive zooms, magnifying the dashed squares in the preceding panels (a)-(g). Except for the first step, all magnifications are ten-fold. Panels (i)-(l) show a selection of further patterns which in this case are not successive zooms. In all panels, values of $c$ are sampled on a regular $2000 \times 2000$ grid, and axes labels indicate values of the real and imaginary part of $c$ in the outermost corners. The gray scales are based on $\log _{10}\left(n_{\text {div }}\right)$, where lighter gray indicates higher $n_{\text {div }}$. The scales are adjusted for each panel to optimize the contrast. All panels are magnified in Figs. 1-12 in the supplementary material. 
No. As can be seen from an iterative zoom across several orders of magnitude, this is not the case [Fig. 3]. At all length scales in the complex plane, $\mathcal{B}$ divides $\mathcal{A}$ into smaller and smaller subsets. To describe this fragmentation, we coin the terminology of ridges of instability which separate lakes of stability. We can observe that each lake of stability contains $c$ values leading to exclusively one of the five states of synchronization. Sometimes apparently adjacent areas of different non-gray colors at one magnification in Fig. 3 might suggest that a lake of stability includes different types of synchronization. However, a further zoom always reveals that these areas of different colors are actually separated by ridges of instability and therefore belong to different lakes of stability. In their entirety, the lakes of stability constitute $\mathcal{A}$. The ridges of instability belong to $\mathcal{B}$, which furthermore includes the overall enclosure of $\mathcal{A}$. In analogy to what we observe for the upper principal period-3 bulb in Fig. 2(d), we see in Figs. 3(b) and 3(c) that the connections between the main cardioid and higher-period bulbs are obstructed by ridges of instability. We use the upper-left period-5 bulb as example to zoom into smaller and smaller length scales in Figs. 3(c)-3(h). One can appreciate that the boundaries between instability ridges and stability lakes are never simple lines. Instead these boundaries form fractal patterns. While these patterns show some similarities across length scales, a rich repertoire of diverse shapes is found on different scales [Figs. 3(a)-3(h)] and at different sites in the complex plane [Figs. 3(i)-3(l)]. Patterns never seem to be replicated identically at different scales, and $\mathcal{A}$ is therefore not strictly self-similar. In more qualitative terms, one may say that details of the set $\mathcal{A}$ seem less filigree than those of the Mandelbrot set. On the other hand, $\mathcal{A}$ seems to be even fuller of variety.

Oftentimes ridges of instability resemble octopus tentacles [Figs. 3(c) and 3(e)-3(g)]. Two vertically oriented and comparably straight tentacle-like ridges enclose a purple lake in the upper left of Fig. 3(e). The lower part of this purple lake is filled by instabilities, which in turn are perforated by countless purple and yellow stability lakes. The light gray color dominating the upper part of these instabilities indicates that for these $c$ values $F_{c}$ undergoes particularly long transients before the iterates eventually diverge to infinity. In this light gray region, we furthermore see cavities that resemble the main bulbs of the Mandelbrot set. These bulbs are segregated by tentacle-like structures, which get thinner and thinner towards the connections of the bulbs with the major purple lake. A further peculiar phenomenon is seen at the rim of the main period-2 bulb [Fig. 3(i)]. The way in which the instabilities penetrate this bulb might be compared to a lava flow overrunning a coastline. This flow also buries most of the period-6 bulb adjacent to the main period-2 bulb in the upper right of Fig. 3(i). Similar instabilities enter the main period-4 bulb from its left side [Fig. 3(j)]. Here stability lakes with a high degree of self-similarity are encapsulated by the instabilities. This region of $\mathcal{A}$ is dominated by full desynchronization such that all stability lakes are depicted in red in Fig. 3(j).

In Fig. 3(k) our attention is at first caught by the pairs of bean-shaped blue and purple lakes of stability. Opposite to the blue lakes we find fountains of rays. These rays contain the aforementioned pairs of blue and purple lakes of stability which grow in size upon increasing distance from the fountain centers. At first glance, some pairs of bean-shaped blue and purple lakes seem to be completely surrounded by instability, isolating them from any other formation. A closer look, however, reveals that this is not the case. What appears to be the connections of blue and purple lakes is also the origin of a stream filled with lakes that are smaller compared to the lakes from which they originate from. These springs grow and unite with other springs, such that the part of $\mathcal{A}$ displayed in Fig. 3(k) does not contain any discernible formations completely surrounded by instability. In other regions of $\mathcal{A}$, however, we do find isolated formations of lakes. Consider for example the groups close to the diagonal of Fig. 3(h). To localize these groups, note that four of them are included in their entirety in Fig. 3(h), while two and one extend beyond the left and right margin of this panel, respectively. Each formation is constituted by a multitude of lakes and can be delineated as a distinct group since it is completely enclosed by ridges of instability. Further examples of isolated groups can be seen in the dark gray regions in Fig. 3(c) and in the periphery of a period-8 bulb [Fig. 3(1)]. To the best of our observation, in none of these cases there are channels of stability which would cross through the instability ridges to establish a connection to these isolated formations. Hence, the set $\mathcal{A}$ is not connected, which distinguishes it from the Mandelbrot set which is known to be connected (see Ref. 54 and references therein).

After ruling out the global connectivity of $\mathcal{A}$, an important question remains. Are different lakes of stability connected locally? In particular, can we take a path in the complex plane leading from one type of synchronization to another one without passing across a ridge of instability? As a first part of the answer, we note that we never observe line-shaped boundaries with zero broadness and non-zero length which would separate areas formed by $c$ values leading to different types of synchronization. Based on this observation, we conclude that each lake of synchronization contains only $c$ values leading to exclusively one state of synchronization. However, connections between stability lakes might be established at points. To address this question, we take a closer look at swirls, examples of which can already be seen in Fig. 3. To analyze these swirls in more detail, we start in Fig. 4(a) with an example taken from the boundary of the main period-2 bulb of $\mathcal{A}$, and continue with iterative zooms into a prominent swirl of four tentacle-like instability ridges [Figs. 4(b)-4(d)]. Swirls of seven ridges are enclosed between two of the major ridges. Like indicated in the context of Fig. $3(\mathrm{j})$, this region of $\mathcal{A}$ is dominated by full desynchronization. Therefore, the stability lakes in Figs. 4(a)-4(d) are depicted in red. This is different in the example shown in Figs. 4(e)-4(h), which is taken from the border of an instability ridge at the entrance of the main period-3 bulb of $\mathcal{A}$. Here, two stability lakes 

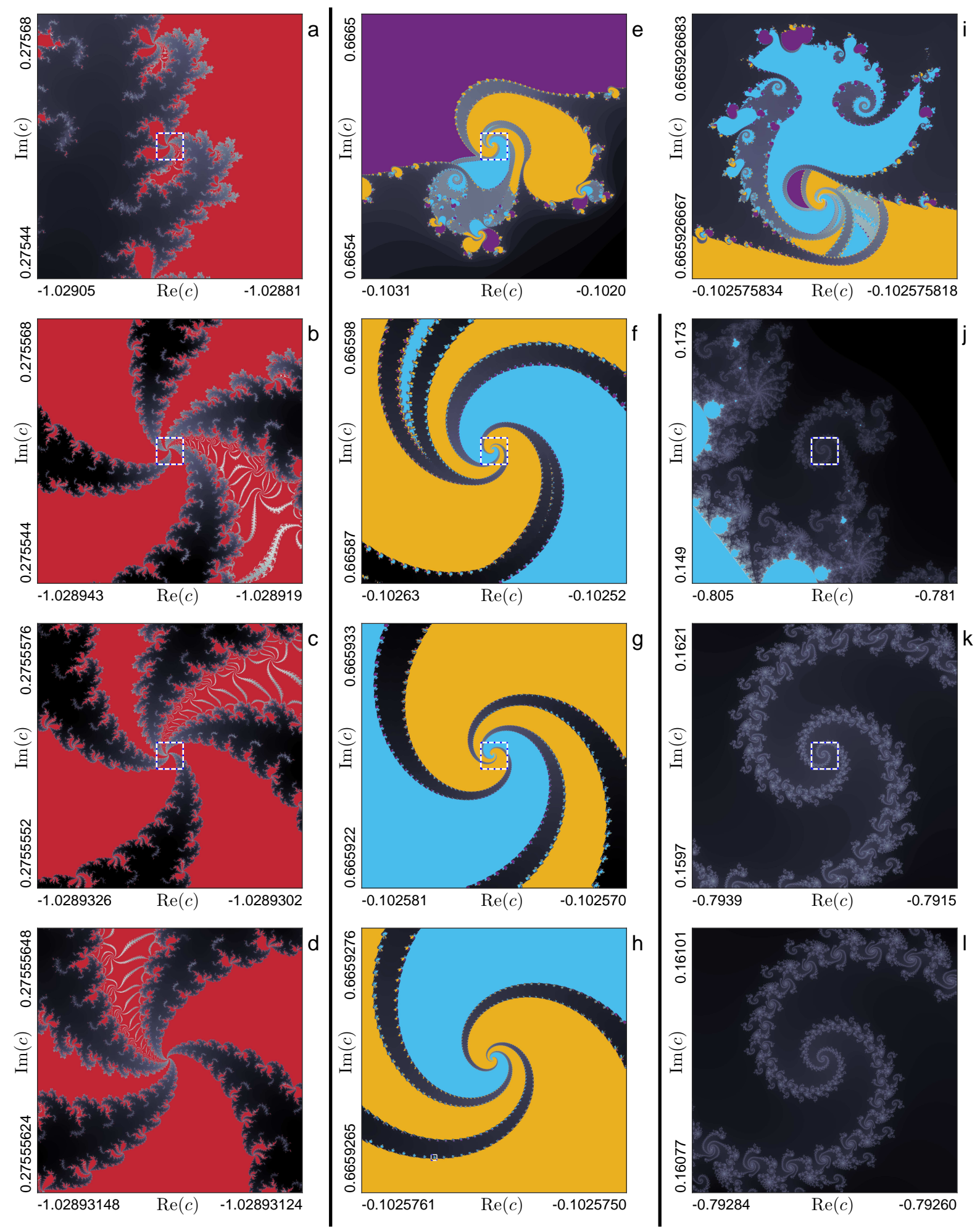

FIG. 4. Octopus's garden. (a)-(d) Same as Fig. 3, here highlighting a spiral-shaped swirl in $\mathcal{A}$. Panel (b)-(d) are successive tenfold zooms, magnifying the dashed squares in the preceding panels (a)-(c). Recall that the gray scales are adjusted for each panel to optimize the contrast. Without this adjustment, the instability ridges in panel (d) would be entirely light grey. (e)-(h) Same as panels (a)-(d), here showing a further example for a swirl. Panel (i) shows a magnification of the square in panel (h), corresponding to a 69-fold zoom. (j)-(l) Analogous to (a)-(h) showing a swirl for the Mandelbrot set. Accordingly, while panels (a)-(h) show results for the network of four coupled quadratic maps $F_{c}$, panels $(\mathrm{j})$-(l) are obtained for an individual quadratic map $f_{c}$. Graphs are drawn like in Figs. 2(a) and 2(c) but without distinguishing periodicity. Instead all $c$ values belonging to the Mandelbrot are plotted in blue. All panels are magnified in Figs. 13-24 in the supplementary material. 


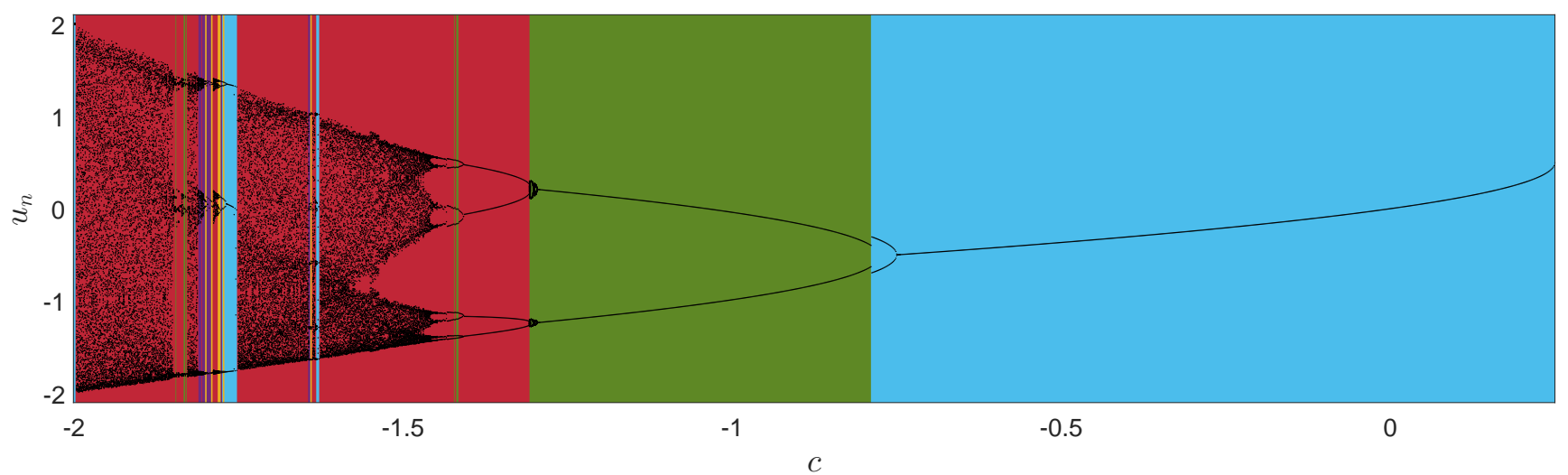

FIG. 5. Real-valued $c$ lead to both periodic and aperiodic dynamics of the network of four coupled quadratic maps $F_{c}$ covering all states of synchronization. Asymptotic states of $u_{n}$ in dependence on $c$ varied within $\mathcal{I}$ in steps of 0.013 [same step size as in Figs. 2(a), 2(b) and 3(a)]. For $c$ leading to a dynamics with period- $m$, dots show the $m$ iterates constituting this cycle. Accordingly, dynamics of period $m$ can be identified by $m$ branches at the corresponding $c$ value. In contrast, for $c$ leading to aperiodic dynamics, the iterates are not constrained to such branches, and the dots show the last 100 iterates of $u_{n}$ prior the $n_{\max }$-th iterate. The colors indicate which state of synchronization the network attains in dependence on $c$. Blue: full synchronization; yellow: within-pair synchronization; green: across-pair synchronization; purple: chimera state; red: full desynchronization (same color code as in Figs. 3-4). Analogous results to those shown here for $U_{c}$ are obtained for the other three maps (not shown). At the right transition from full synchronization (blue) to across-pair synchronization (green), the fully synchronized period-2 motion of all four maps $\left(U_{c}=P_{c}=V_{c}=Q_{c}\right)$ loses stability. Two distinct period-2 orbits (for example $U_{c}=P_{c} \neq V_{c}=Q_{c}$ ) arise instead. This explains discontinuities at such transitions in this bifurcation diagram of $U_{c}$.

containing $c$ values leading to within-pair synchronization and one stability lake containing $c$ values leading to full synchronization jointly spiral into the swirl center. Furthermore, a magnification into one of the separating instability ridges reveals stability lakes of chimera states [Fig. 4(i)].

What is in the center of such swirls? To address this question for $\mathcal{A}$, we at first briefly look back at the Mandelbrot set. An example for a spiral-shaped swirl in the Mandelbrot set is shown in Figs. 4(j)-4(l). The periphery of the Mandelbrot set contains countless miniature approximate copies of itself [Figs. 4(j)]. However, no such miniature Mandelbrot set is located in the swirl center. Due to the asymptotic self similarity at the center of the spiral, successive zooms solely seem to result in a rotation. No matter how much one increases the zoom, one would continue to see the same self similar spiral. The center cannot be resolved ${ }^{55}$. Nonetheless, since the Mandelbrot set is connected, we know that the swirl center must belong to the Mandelbrot set. Otherwise, it would be impossible to pass from one spiral arm to the other, which would contradict with the set's connectedness. We conjecture that also the swirl centers in $\mathcal{A}$ are point-like. In other words, we assume that they are not formed by a stability lake or instability ridge of non-zero area. However, for $\mathcal{A}$ we cannot use connectedness to conclude to the nature of this point. The swirl centers can belong to $\mathcal{A}$ or to $\mathcal{B}$. One might speculate that this depends on whether or not all lakes of stability which approach a particular swirl center are of the same type of synchronization. If they all are of the same type, such as in Figs. 4(a)-4(d), the swirl center might belong to $\mathcal{A}$ and show that same type of synchronization. If the lakes contain different types of synchronization, such as in Figs. 4(e)-4(h), they might be separated by the swirl center belonging to $\mathcal{B}$. However, these remain conjectures, and a definite answer as to whether swirl centers can be used to pass from one type of synchronization to another without leaving $\mathcal{A}$ is left for future studies.

\section{THE PECULIARITY OF THE REAL AXIS}

The dynamics of $F_{c}$ for parameters taken from the real axis, i.e. $c \in \mathbb{R}$, merit additional consideration (Fig. 5). First, we recall that the Mandelbrot set as well as $\mathcal{A}$ are symmetric around the real axis and we note that both include the interval $\mathcal{I}=[-2,0.25] \subset \mathbb{R}$ [see again Figs. 2(a) and 2(b)]. For all $c \in \mathcal{I}$, both $f_{c}$ and $F_{c}$ show bounded behaviour, whereas they both diverge for all $c$ which are real-valued but outside of $\mathcal{I}$. At the interval limits $c=-2$ and $c=0.25$, the map $f_{c}$ converges to the fixed points 2 and 0.5 , respectively. The network shows the same behaviour, and all four maps converge to 2 for $F_{c=-2}$, and to 0.5 for $F_{c=0.25}$. In consequence, at both limits of $\mathcal{I}$, the network $F_{c}$ enters into a fully synchronized state. Across the $c \in \mathcal{I}, F_{c}$ attains all five states of synchronization (Fig. 5). Hence, all lakes of stability which include subintervals of $\mathcal{I}$ are connected via the points at the limits of their respective subintervals. 
Therefore, going along $\mathcal{I}$, it is possible to switch between all states of synchronization without crossing any ridge of instability. While we cannot answer yet whether swirl centers can be used to pass from one type of synchronization to another without leaving $\mathcal{A}$, we see that this passage is possible taking the simple path offered by $\mathcal{I}$.

A further important aspect is the existence of bounded aperiodic solutions of $F_{c}$ for some of the $c \in \mathcal{I}$. It is known that decreasing the parameter $c$ along $\mathcal{I}$, leads to a period-doubling bifurcation scenario for the individual map $f_{c}$. In Fig. 5 we use the example of $U_{c}$ to show that the maps still exhibit a bifurcation scenario also when they are coupled to form the network $F_{c}$. Their bifurcation diagrams are different from the one of $f_{c}$, and the diagrams' fine structure depends on the coupling values $C_{\mathrm{w}}$ and $C_{\mathrm{a}}$ (see also Ref. 39). What matters here is that for $c \in \mathcal{I}$ we find both periodic and aperiodic solutions of $F_{c}$. For the coupling values used here, the fully desynchronized state, across-pair synchronization and chimera states are attained for both periodic and aperiodic dynamics. In contrast, the fully synchronized state and within-pair synchronization are only found in the periodic windows of the bifurcation diagram shown in Fig. 5.

Conversely, we found no evidence for bounded aperiodic solutions of $F_{c}$ for any $c \notin \mathcal{I}$, i.e. for parameter values away from this subinterval of the real axis. Recall that for real-valued $c \notin \mathcal{I}$, the network always diverges to infinity. Very rarely we found complex-valued $c \notin \mathcal{I}$ for which after $n_{\max }$ iterations the network was still bounded and had not yet entered into any periodic cycle. However, detailed inspection of these particular cases always showed that transients were still ongoing. When we observed the dynamics beyond $n_{\max }$ iterations, the transients continued along contracting or expanding patterns in the complex plane in a slow manner, suggesting that the dynamics would eventually settle down to a periodic cycle or diverge, respectively. A reliable distinction between long transients in bounded or diverging solutions, cycles of very high but finite period, and aperiodic solutions, if the latter exist for $c \notin \mathcal{I}$, will require a refined methodology and is left for future studies. Therefore, while Fig. 5 shows that $\mathcal{I} \subset \mathcal{A}$, and for some $c \in \mathcal{I}$ the network $F_{c}$ shows bounded aperiodic dynamics, we can only say that we found no evidence for such dynamics for parameters $c$ with $c \in \mathcal{A}$ but $c \notin \mathcal{I}$.

\section{DISCUSSION AND OUTLOOK}

The present study establishes a link between the dynamics of chimera states and the geometry of fractal structures in the complex plane. First links between chimeras and fractals were drawn already in Refs. 21-23. These studies showed that the set of initial conditions leading to chimeras ${ }^{21,22}$ or sub-chimeras ${ }^{23}$ form riddled and fractal basins of attraction for networks of Rössler dynamics ${ }^{22}$, Henon maps ${ }^{21}$, logistic maps ${ }^{23}$, and sine-squared maps ${ }^{23}$ (see also Ref. 56). The spaces of basins of attraction are high-dimensional, and one can only inspect projections to two dimensions. In contrast, our fractal set $\mathcal{A}$ resides in the complex plain and can therefore readily be displayed and explored. We furthermore note that the term fractal is used to refer to certain hierarchical connectivity architectures in networks generating chimera networks (e.g. Refs. 28, 57-61). However, this notion of fractal connectivity is unrelated from the way in which the term fractal is used in Refs. 21-23 and in the present study. Refs. 62 and 63 studied ring networks of $N$ locally coupled quadratic maps for which the parameters $c$ were varied independently for each map yielding a spatial Mandelbrot set of dimensionality $2 N$. This high dimensionality again only allows one to inspect projections to subspaces, and Refs. 62 and 63 did not analyze the dynamics of the coupled maps with regard to their synchronization.

There are several important advances achieved by the present study with regard to our previous work, ${ }^{39}$ in which we used two large populations of each 100 quadratic maps $f_{c}$. In the main part of Ref. 39, we studied the network's dynamics for a single real-valued $c=-1.8 \in \mathcal{I}$ using random real-valued initial conditions. Upon variation of the coupling strengths within and across populations, the network showed a plentitude of synchronous versus asynchronous, periodic versus aperiodic and symmetric versus symmetry broken dynamics. It furthermore exhibited multistability by settling to distinct dynamics for different realizations of the initial conditions or by switching intermittently between distinct dynamics for the same realization. However, since we used a real-valued $c$ and real-valued initial conditions, the network dynamics remained real-valued throughout the main part of Ref. 39. Only as a brief outlook, this previous work studied for which complex valued $c$, the network iterates remained bounded or diverged. Results already showed that the capacity of the quadratic map $f_{c}$ to generate fractal structures in the complex plane is inherited by networks of coupled quadratic maps. However, beyond the classification into bounded and divergent behaviour, Ref. 39 paid no further attention to the network's dynamics. This is achieved in the present study, by detecting different states of synchronization of the network's iterates in the complex plane (see again Fig. 1 and table I). The most important finding, in our view, is that mapping the different states of synchronization of $F_{c}$ as a function of its parameter $c$ in the complex plane leads to fractal structures with an additional level of complexity. The set $\mathcal{A}$, defined by the set of $c$ for which $F_{c}$ remains bounded, is divided into a presumably infinite number of subsets. We here coined the terms of lakes of stability which are enclosed by ridges of instability. To the best of our observation, each lake contains exactly one state of synchronization (see again Fig. 3-4). Arbitrarily small changes in $c$ can be decisive for whether the network iterates diverge or remain bounded and determine whether bounded solutions get fully synchronized, 

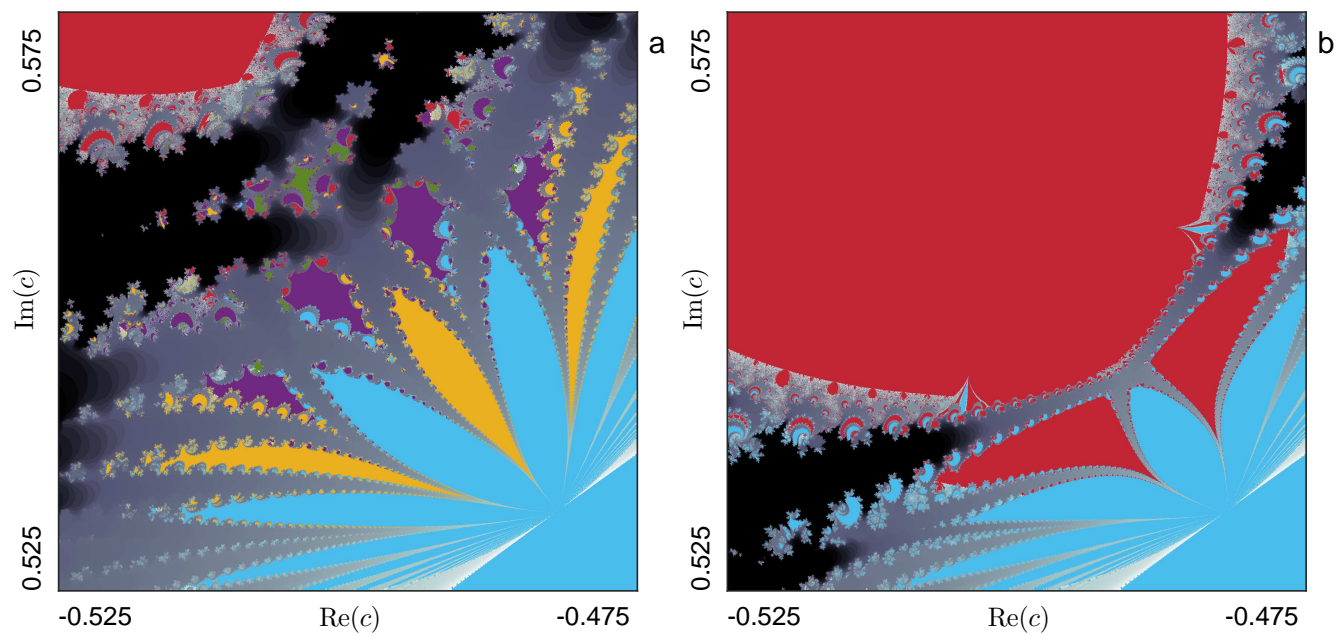

FIG. 6. Impact of coupling strengths and an even simpler network. (a) Same as Fig. $3(\mathrm{c})$ but here for $C_{\mathrm{W}}=0.02$ and $C_{\mathrm{a}}=0.0025$. Accordingly, panel (a) shows results for the network of four coupled quadratic maps $F_{c}$. Results shown in panel (b) are obtained for the same area as in (a) but for a network reduced to a single pair of two quadratic maps $U_{c}$ and $V_{c}$ coupled with $C_{\mathrm{W}}=0.01$. We observe synchronization $U_{c}=V_{c}$ (blue), desynchronization $U_{c} \neq V_{c}$ (red), or divergence to infinity (gray).

fully desynchronized, or enter into some partially synchronized state, including a chimera state. Nonetheless, the only path in the complex plane for which we can confirm that it allows one to pass between all states of synchronization without crossing an instability is given by the real-valued $c \in \mathcal{I}=[-2,0.25]$.

A further important difference between the present study and our previous work ${ }^{39}$, originates from the way in which we can define the initial conditions of these networks. In the main part of Ref. 39, which was solely dealing with real-valued dynamics, we followed earlier work of ours ${ }^{64-68}$ and used random initial conditions. However, as explained in the following, random initial conditions do not allow us to generate fractal structures in the complex plane. We at first briefly note that the $c$ values forming the Mandelbrot set are all contained in the closed disk of radius 2 around the origin of the complex plane. For all $c$ in this disk, the map $f_{c}\left(z_{n=0}\right)$ diverges for $\left|z_{n=0}\right|>2$. Therefore, any initial conditions $\left|z_{n=0}\right|>2$ are directly discarded, and the following considerations are restricted to initial conditions $\left|z_{n=0}\right| \leq 2$. For a given value of $c$, it depends on $z_{n=0}$ whether the individual map $f_{c}$ diverges or remains bounded. The boundaries between diverging and bounded behaviour of $f_{c}$ in the domain of complex-valued initial conditions $z_{n=0}$ defines Julia sets ${ }^{40,41}$. In consequence, the question if the iterates of $f_{c}$ or $F_{c}$ remain bounded for a certain $c$, cannot be answered without specifying the initial conditions. That is the reason why the set $\mathcal{A}$, which is defined from the boundedness of $F_{c}$, cannot be defined for random initial conditions.

Our approach to use the first $2 N$ iterates of the uncoupled map $f_{c}$ started at $z_{0}=0$ as initial conditions for the two populations of each $N$ maps is straightforward, but remains of course somewhat arbitrary. In fact, for high $N$ it leads to a different problem. For many values of $c$ outside of the Mandelbrot set, the map $f_{c}$ diverges quickly and leaves the limits of floating point numbers after a few dozens of iterations. For these $c$ values it was therefore impossible to generate 200 finite-valued iterates to be used as initial conditions for the $2 N=200$ maps used in Ref. 39. For this reason, we restricted this previous study to $c$ values inside the Mandelbrot set. By definition of this set, the map $f_{c}$ never diverges for these $c$ and initial conditions can, therefore, always be defined from the first 200 iterates. In consequence, in Ref. 39 we could only study the additional fractal structure generated inside the Mandelbrot set. Since we here only use four maps, this limitation does not apply. The iterates of $f_{c}$ started at $z_{n=0}=0$ never leave the limits of floating point numbers within the first $n=4$ iterates. These first iterates can always be used as initial conditions for $F_{c}$. In the current study, we can, therefore, assess the full set $\mathcal{A}$ without restricting it a priori to the inside of the Mandelbrot set.

We concluded in Ref. 39 that the high dimensionality of two populations of each 100 quadratic maps leads to the additional complexity in the fractal patterns described in this previous work. The present study, however, shows that this complexity is already observed for the minimal two-population network of two pairs of two quadratic maps. In fact, upon variation of the coupling strengths we can generate a whole family of fractal sets $\mathcal{A}\left(C_{\mathrm{w}}, C_{\mathrm{a}}\right)$. As one example, in Fig. 6(a) we revisit the area already inspected in Fig. 3(c) but now using $C_{\mathrm{w}}=0.02$ and $C_{\mathrm{a}}=0.0025$ instead of $C_{\mathrm{w}}=0.01$ and $C_{\mathrm{a}}=0.0025$. The change of the coupling strengths leads to a major reshaping of the instability 
ridges and enclosed stability lakes. A study of the coupling strengths' influence should also test for the existence of across-pair chimera states (e.g. $U_{c}=P_{c} \neq V_{c} \neq Q_{c} \neq U_{c}$ ) or triplet-singlet states ${ }^{48}$ (e.g. $U_{c}=P_{c}=V_{c} \neq Q_{c}$ ).

Can we observe fractal boundaries of synchronization for even smaller networks? Yes, as we can see in Fig. 6(b), a single pair of two coupled quadratic maps is already sufficient. To illustrate this, we set $C_{\mathrm{w}}=0.01$ and $C_{\mathrm{a}}=0$ such that the network $F_{c}$ falls apart into two mutually independent pairs of maps, of which we consider only the pair $U_{c}$ and $V_{c}$. Since we have only two maps, there are only two possible bounded states. Either the two maps synchronize or they do not [Fig. 6(b)]. Despite this reduction in the diversity of the dynamics, the fractal patterns generated by just two maps seem of similar complexity as the ones generated by two pairs of two maps.

\section{SUPPLEMENTARY MATERIAL}

See supplementary material consisting of Supplementary Figs. 1-12 for enlarged displays of the 12 panels of Fig. 3 and Supplementary Figs. 13-24 for enlarged displays of the 12 panels of Fig. 4.

\section{ACKNOWLEDGMENTS}

We are grateful to Anaïs Espinoso, Christian Rummel and Yuanzhao Zhang for useful discussions on the manuscript.

\section{Appendix}

\begin{tabular}{|l|l|l|}
\hline$\#$ & value & Fig. 1 \\
\hline \hline$c_{1}$ & $-0.25550551+0.63328328 i$ & $(\mathrm{a})$ \\
$c_{2}$ & $-0.11431431+0.65605606 i$ & (b) \\
$c_{3}$ & $-0.12972973+0.65500501 i$ & (c) \\
$c_{4}$ & $-0.11746747+0.66306306 i$ & (d) \\
$c_{5}$ & $-0.10665959+0.67261359 i$ & (e) \\
$c_{6}$ & $-0.11116116+0.67392392 i$ & (f) \\
$c_{7}$ & $+0.37579580+0.17822823 i$ & (g) \\
$c_{8}$ & $-0.10665959+0.67261349 i$ & (h) \\
\hline
\end{tabular}

TABLE II. Values of the parameter $c$ used to generate the examples shown in Fig. 1 with the respective panel indicated in the last column. We specify the values with eight significant digits. Since arbitrarily small changes of $c$ can lead to a qualitatively different asymptotic dynamics, one needs the exact values at double floating point precision to reproduce Fig. 1. These values are provided along with the source codes to run our computations at the electronic library repository of the Universitat Pompeu Fabra $^{69}$.

\section{DATA AVAILABILITY STATEMENTS}

The source codes used for the computations of this study are openly available in the repository used by the Universitat Pompeu Fabra: https://doi.org/10.34810/data49. See Ref. 69.

\section{REFERENCES}

${ }^{1}$ V. V. Astakhov, V. S. Anishchenko, and A. V. Shabunin, "Controlling spatiotemporal chaos in a chain of the coupled logistic maps," IEEE Transactions on Circuits and Systems I: Fundamental Theory and Applications 42, 352-357 (1995).

${ }^{2}$ V. S. Anishchenko, T. E. Vadivasova, G. I. Strelova, and A. S. Kopeikin, "Chaotic attractors of two-dimensional invertible maps," Discrete Dynamics in Nature and Society 2, 249-256 (1998).

${ }^{3}$ V. Astakhov, A. Shabunin, A. Klimshin, and V. Anishchenko, "In-phase and antiphase complete chaotic synchronization in symmetrically coupled discrete maps," Discrete Dynamics in Nature and Society 7, 215-229 (2002).

${ }^{4}$ T. E. Vadivasova, G. I. Strelkova, S. A. Bogomolov, and V. S. Anishchenko, "Correlation analysis of the coherence-incoherence transition in a ring of nonlocally coupled logistic maps," Chaos 26, 093108 (2016).

${ }^{5}$ S. Bogomolov, G. Strelkova, E. Schöll, and V. Anishchenko, "Amplitude and phase chimeras in an ensemble of chaotic oscillators," Technical Physics Letters 42, 765-768 (2016). 
${ }^{6}$ S. A. Bogomolov, A. V. Slepnev, G. I. Strelkova, E. Schöll, and V. S. Anishchenko, "Mechanisms of appearance of amplitude and phase chimera states in ensembles of nonlocally coupled chaotic systems," Commun. Nonlinear Sci. Numer. Simul. 43, 25-36 (2017).

${ }^{7}$ G. I. Strelkova, T. E. Vadivasova, and V. S. Anishchenko, "Synchronization of chimera states in a network of many unidirectionally coupled layers of discrete maps," Regular and Chaotic Dynamics 23, 948-960 (2018).

${ }^{8}$ E. Rybalova, T. Vadivasova, G. Strelkova, V. Anishchenko, and A. Zakharova, "Forced synchronization of a multilayer heterogeneous network of chaotic maps in the chimera state mode," Chaos 29, 033134 (2019).

${ }^{9} \mathrm{M}$. Winkler, J. Sawicki, I. Omelchenko, A. Zakharova, V. Anishchenko, and E. Schöll, "Relay synchronization in multiplex networks of discrete maps," EPL (Europhysics Letters) 126, 50004 (2019).

${ }^{10}$ N. Semenova, A. Zakharova, E. Schöll, and V. Anishchenko, "Does hyperbolicity impede emergence of chimera states in networks of nonlocally coupled chaotic oscillators?" EPL (Europhysics Letters) 112, 40002 (2015).

${ }^{11}$ N. I. Semenova, E. V. Rybalova, G. I. Strelkova, and V. S. Anishchenko, "coherence-incoherence transition in ensembles of nonlocally coupled chaotic oscillators with nonhyperbolic and hyperbolic attractors," Regular and Chaotic Dynamics 22, 148-162 (2017).

${ }^{12}$ N. Semenova, G. Strelkova, V. Anishchenko, and A. Zakharova, "Temporal intermittency and the lifetime of chimera states in ensembles of nonlocally coupled chaotic oscillators," Chaos 27, 061102 (2017).

${ }^{13}$ A. Bukh, E. Rybalova, N. Semenova, G. Strelkova, and V. Anishchenko, "New type of chimera and mutual synchronization of spatiotemporal structures in two coupled ensembles of nonlocally interacting chaotic maps," Chaos 27, 111102 (2017).

${ }^{14}$ E. Rybalova, G. Strelkova, E. Schöll, and V. Anishchenko, "Relay and complete synchronization in heterogeneous multiplex networks of chaotic maps," Chaos 30, 061104 (2020).

${ }^{15}$ E. Rybalova, G. Strelkova, and V. Anishchenko, "Impact of sparse inter-layer coupling on the dynamics of a heterogeneous multilayer network of chaotic maps," Chaos, Solitons \& Fractals 142, 110477 (2021).

${ }^{16}$ I. A. Shepelev, A. V. Bukh, G. I. Strelkova, T. E. Vadivasova, and V. S. Anishchenko, "Chimera states in ensembles of bistable elements with regular and chaotic dynamics," Nonlinear Dynamics 90, 2317-2330 (2017).

${ }^{17}$ I. A. Shepelev, A. Bukh, T. E. Vadivasova, V. S. Anishchenko, and A. Zakharova, "Double-well chimeras in 2d lattice of chaotic bistable elements," Commun. Nonlinear Sci. Numer. Simul. 54, 50-61 (2018).

${ }^{18}$ A. Bukh, E. Schöll, and V. Anishchenko, "Synchronization of spiral wave patterns in two-layer 2d lattices of nonlocally coupled discrete oscillators," Chaos 29, 053105 (2019).

${ }^{19}$ Y. Kuramoto and D. Battogtokh, "Coexistence of coherence and incoherence in nonlocally coupled phase oscillators." Nonlinear Phenom. Complex Syst. 5, 380-385 (2002).

${ }^{20}$ D. M. Abrams and S. H. Strogatz, "Chimera states for coupled oscillators," Phys. Rev. Lett. 93, 174102 (2004).

${ }^{21}$ V. Santos, J. D. Szezech, A. M. Batista, K. C. Iarosz, M. S. Baptista, H. P. Ren, C. Grebogi, R. L. Viana, I. L. Caldas, Y. L. Maistrenko, and J. Kurths, "Riddling: Chimeras dilemma," Chaos 28, 081105 (2018).

${ }^{22}$ V. dos Santos, F. S. Borges, K. C. Iarosz, I. L. Caldas, J. Szezech, R. L. Viana, M. S. Baptista, and A. M. Batista, "Basin of attraction for chimera states in a network of Rössler oscillators," Chaos 30, 083115 (2020).

${ }^{23}$ Y. Zhang, Z. G. Nicolaou, J. D. Hart, R. Roy, and A. E. Motter, "Critical switching in globally attractive chimeras," Phys. Rev. X 10, $011044(2020)$.

${ }^{24}$ I. Omelchenko, Y. Maistrenko, P. Hövel, and E. Schöll, "Loss of coherence in dynamical networks: spatial chaos and chimera states," Phys. Rev. Lett. 106, 234102 (2011).

${ }^{25}$ I. Omelchenko, B. Riemenschneider, P. Hövel, Y. Maistrenko, and E. Schöll, "Transition from spatial coherence to incoherence in coupled chaotic systems," Phys. Rev. E 85, 026212 (2012).

${ }^{26}$ Y. Zhang and A. E. Motter, "Mechanism for strong chimeras," arXiv preprint arXiv:2101.12230 (2021).

${ }^{27}$ I. Korneev, V. Semenov, A. Slepnev, and T. Vadivasova, "Complete synchronization of chaos in systems with nonlinear inertial coupling," Chaos, Solitons \& Fractals 142, 110459 (2021).

${ }^{28}$ A. zur Bonsen, I. Omelchenko, A. Zakharova, and E. Schöll, "Chimera states in networks of logistic maps with hierarchical connectivities," The European Physical Journal B 91, 65 (2018).

${ }^{29}$ S. Ghosh, A. Zakharova, and S. Jalan, "Non-identical multiplexing promotes chimera states," Chaos, Solitons \& Fractals 106, 56-60 (2018).

${ }^{30}$ P. Chandran, R. Gopal, V. Chandrasekar, and N. Athavan, "Chimera states in coupled logistic maps with additional weak nonlocal topology," Chaos 29, 053125 (2019).

${ }^{31}$ T. Vadivasova, A. Slepnev, and A. Zakharova, "Control of inter-layer synchronization by multiplexing noise," Chaos 30, 091101 (2020).

${ }^{32}$ L. Khaleghi, S. Panahi, S. N. Chowdhury, S. Bogomolov, D. Ghosh, and S. Jafari, "Chimera states in a ring of map-based neurons," Physica A: Statistical Mechanics and its Applications 536, 122596 (2019).

${ }^{33}$ C. R. Nayak and N. Gupte, "Chimera states in coupled sine-circle map lattices," in AIP Conference Proceedings, Vol. 1339 (2011) pp. $172-180$.

${ }^{34}$ J. Singha and N. Gupte, "Spatial splay states and splay chimera states in coupled map lattices," Phys. Rev. E 94, 052204 (2016).

${ }^{35}$ J. Singha and N. Gupte, "Chimera states in coupled map lattices: Spatiotemporally intermittent behavior and an equivalent cellular automaton," Chaos 30, $113102(2020)$.

${ }^{36}$ J. D. Hart, D. C. Schmadel, T. E. Murphy, and R. Roy, "Experiments with arbitrary networks in time-multiplexed delay systems," Chaos 27, 121103 (2017).

${ }^{37}$ A. M. Hagerstrom, T. E. Murphy, R. Roy, P. Hövel, I. Omelchenko, and E. Schöll, "Experimental observation of chimeras in coupled-map lattices," Nat. Phys. 8, 658-661 (2012).

${ }^{38}$ D. Dudkowski, Y. Maistrenko, and T. Kapitaniak, "Different types of chimera states: An interplay between spatial and dynamical chaos," Phys. Rev. E 90, 032920 (2014).

${ }^{39}$ R. G. Andrzejak, G. Ruzzene, E. Schöll, and I. Omelchenko, "Two populations of coupled quadratic maps exhibit a plentitude of symmetric and symmetry broken dynamics," Chaos 30, 033125 (2020).

${ }^{40}$ P. Fatou, "Sur les substitutions rationnelles," Comptes Rendus de l'Académie des Sciences de Paris 164, 806-808 (1917).

${ }^{41}$ G. Julia, "Mémoire sur la permutabilité des fractions rationnelles," in Annales scientifiques de l'École Normale Supérieure, Vol. 39 (1922) pp. 131-215.

${ }^{42}$ R. Brooks and J. P. Matelski, "The dynamics of 2-generator subgroups of psl (2, c)," in Riemann surfaces and related topics: Proceedings of the 1978 Stony Brook Conference, Ann. of Math. Stud, Vol. 97 (1981) pp. 65-71. 
${ }^{43}$ B. B. Mandelbrot, "Fractal aspects of the iteration of $z \lambda z(1-z)$ for complex $\lambda$ and $z$," Annals of the New York Academy of Sciences 357, 249-259 (1980).

${ }^{44}$ A. Douady, J. H. Hubbard, and P. Lavaurs, "Etude dynamique des polynômes complexes," Publ. Math. Orsay , 2/4 (1984).

${ }^{45}$ P. Ashwin and O. Burylko, "Weak chimeras in minimal networks of coupled phase oscillators," Chaos 25, 013106 (2015).

${ }^{46}$ F. Böhm, A. Zakharova, E. Schöll, and K. Lüdge, "Amplitude-phase coupling drives chimera states in globally coupled laser networks," Phys. Rev. E 91, 040901 (2015).

${ }^{47}$ M. J. Panaggio, D. M. Abrams, P. Ashwin, and C. R. Laing, "Chimera states in networks of phase oscillators: the case of two small populations," Phys. Rev. E 93, 012218 (2016).

${ }^{48}$ J. D. Hart, K. Bansal, T. E. Murphy, and R. Roy, "Experimental observation of chimera and cluster states in a minimal globally coupled network," Chaos 26, 094801 (2016).

${ }^{49}$ J. Wojewoda, K. Czolczynski, Y. Maistrenko, and T. Kapitaniak, "The smallest chimera state for coupled pendula," Sci. Rep. 6 (2016).

${ }^{50}$ Y. Maistrenko, S. Brezetsky, P. Jaros, R. Levchenko, and T. Kapitaniak, "Smallest chimera states," Phys. Rev. E 95, 010203 (2017).

${ }^{51}$ P. Ebrahimzadeh, M. Schiek, P. Jaros, T. Kapitaniak, S. van Waasen, and Y. Maistrenko, "Minimal chimera states in phase-lag coupled mechanical oscillators," The European Physical Journal Special Topics 229, 2205-2214 (2020).

${ }^{52}$ J. Sharma, I. Tiwari, D. Das, and P. Parmananda, "Chimeralike states in a minimal network of active camphor ribbons," Phys. Rev. E 103, 012214 (2021).

${ }^{53}$ We performed the analysis using Matlab with double floating point precision. The values of the quadratic map are of the order $10^{0}$. The value $\varepsilon_{\text {NUM }}$ is, therefore, just above the resolution of the double floating point precision ${ }^{70}$. Pre-analysis showed this threshold to be optimal in minimizing the impact of numerical roundoff errors.

${ }^{54}$ H.-O. Peitgen, H. Jürgens, and D. Saupe, Chaos and fractals: new frontiers of science (Springer Science \& Business Media, 2006).

${ }^{55}$ For a more detailed discussion of these spirals in the Mandelbrot set, their relation to the corresponding Julia sets and Misiurewicz points see Ref. 54 and references therein.

${ }^{56}$ E. A. Martens, M. J. Panaggio, and D. M. Abrams, "Basins of attraction for chimera states," New Journal of Physics 18, 022002 (2016).

${ }^{57}$ J. Hizanidis, E. Panagakou, I. Omelchenko, E. Schöll, P. Hövel, and A. Provata, "Chimera states in population dynamics: Networks with fragmented and hierarchical connectivities," Phys. Rev. E 92, 012915 (2015).

${ }^{58} \mathrm{~S}$. Ulonska, I. Omelchenko, A. Zakharova, and E. Schöll, "Chimera states in networks of Van der Pol oscillators with hierarchical connectivities," Chaos 26, 094825 (2016).

${ }^{59}$ J. Sawicki, I. Omelchenko, A. Zakharova, and E. Schöll, "Chimera states in complex networks: interplay of fractal topology and delay," The European Physical Journal Special Topics 226, 1883-1892 (2017).

${ }^{60}$ T. Chouzouris, I. Omelchenko, A. Zakharova, J. Hlinka, P. Jiruska, and E. Schöll, "Chimera states in brain networks: Empirical neural vs. modular fractal connectivity," Chaos 28, 045112 (2018).

${ }^{61}$ G. Argyropoulos and A. Provata, "Chimera states with 2d deterministic and random fractal connectivity," Frontiers in Applied Mathematics and Statistics 5, 35 (2019).

${ }^{62}$ P. Liu, "Control and synchronization of Mandelbrot sets in coupled map lattice," Nonlinear Dynamics 73, 299-310 (2013).

${ }^{63}$ D. Wang, S. Liu, Y. Zhao, and C. Jiang, "Control of the spatial Mandelbrot set generated in coupled map lattice," Nonlinear Dynamics 84, 1795-1803 (2016).

${ }^{64}$ R. G. Andrzejak, C. Rummel, F. Mormann, and K. Schindler, "All together now: Analogies between chimera state collapses and epileptic seizures," Sci. Rep. 6, 23000 (2016).

${ }^{65}$ R. G. Andrzejak, G. Ruzzene, and I. Malvestio, "Generalized synchronization between chimera states," Chaos 27, 053114 (2017).

${ }^{66}$ R. G. Andrzejak, G. Ruzzene, I. Malvestio, K. Schindler, E. Schöll, and A. Zakharova, "Mean field phase synchronization between chimera states," Chaos 28, 091101 (2018).

${ }^{67}$ G. Ruzzene, I. Omelchenko, E. Schöll, A. Zakharova, and R. G. Andrzejak, "Controlling chimera states via minimal coupling modification," Chaos 29, 051103 (2019).

${ }^{68}$ G. Ruzzene, I. Omelchenko, J. Sawicki, A. Zakharova, E. Schöll, and R. G. Andrzejak, "Remote pacemaker control of chimera states in multilayer networks of neurons," Phys. Rev. E 102, 052216 (2020).

${ }^{69}$ Open access repository used by the Universitat Pompeu Fabra, https://doi.org/10.34810/data49.

${ }^{70}$ IEEE Standards Committee, "754-2008 IEEE standard for floating-point arithmetic," IEEE Computer Society Std , 1-70 (2008). 